\title{
Arbitrary grounding
}

\author{
Jonas Werner ${ }^{1}$
}

Accepted: 4 July 2021 / Published online: 10 July 2021

(C) The Author(s) 2021

\begin{abstract}
The aim of this paper is to introduce, elucidate and defend the usefulness of a variant of grounding, or metaphysical explanation, that has the feature that the grounds explain of some states of affairs that one of them obtains without explaining which one obtains. I will dub this variant arbitrary grounding. After informally elucidating the basic idea in the first section, I will provide three metaphysical hypotheses that are best formulated in terms of arbitrary grounding in the second section. The third section will be concerned with the relation between arbitrary grounding and non-arbitrary grounding. The fourth section will compare arbitrary grounding to two extant proposals in the literature.
\end{abstract}

Keywords Grounding · Fundamentality · Indeterminism · Modality

\section{Introduction}

Imagine you are standing in front of two identical doughnuts, Dan and Deb, and you wish to eat a doughnut. However, one doughnut is enough for you, you don't want to eat both doughnuts. Although there is no reason to favour one doughnut over the other, most of us manage to grab a doughnut and to happily walk away with it. We seem to have the ability to make arbitrary decisions. Citing reasons surely is a way to provide explanations. In this case, you can explain why you took a doughnut by citing reasons concerning your appetite and your love for sweets. Even if you cannot explain why you took Dan, rather than Deb, your explanation seems to be complete. There is simply no reason to favour Dan over Deb you should cite to complete the

Jonas Werner

jonas.werner@philo.unibe.ch

1 University of Bern, Institute of Philosophy, Länggassstrasse 49a, 3012 Bern, Switzerland 
explanation. The choice between the two doughnuts was not apt to be explained, at least not by giving reasons.

There might also be causal explanations that have this structure. Imagine an ordinary six-sided die that is thrown in a world in which causal determinism fails. Assume that the causal laws in this world determine that when the die lands, it will land on the side that shows a two or on the side that shows a four, but the laws don't determine on which of these two sides the die will land. The die lands on the side that shows a four. Let us assume for the sake of this example that causal laws help to explain particular matters of fact. Given this assumption, it seems to not be completely unexplained that the die shows a four: We can explain why it shows a four, rather than a one, three, five or six. Given that there is no other factor that determined on which side the die lands, it is simply arbitrary, however, that it shows a four and not a two, although it is fully explained that it shows one of these two numbers.

This paper argues that we should also be willing to countenance groundingexplanations that are arbitrary in this sense. To familiarize the reader with the idea, I present a theologically flavoured toy-example of such an arbitrary metaphysical explanation. Let us assume that every non-fundamental fact is grounded in god's will. Let us further assume that god wills there to be ten apples on the tree of knowledge of which exactly one is such that its being eaten leads to the fall. God doesn't decide of a particular one of the ten apples that it is the one that has the fallinducing property, she doesn't care which of the apples has this special property. In this case, it seems to not be unexplained that Anna the apple has the fall-inducing property, but there is no explanation forthcoming to the extent that Anna, rather than some other apple from the tree, is the one that has this property. If there can be arbitrary metaphysical explanations, then god might get away with decreeing that one of the apples be the dangerous one, without decreeing which one.

The underlying idea is that there might be cases in which some facts, the $f f$, explain that one of the states of affairs $g g$ obtains without explaining which one. ${ }^{1}$ Which of the $g g$ obtains is an arbitrary choice. Let us assume that $g$ is the one of the $g g$ that obtains. In such a case I will say that the $f f$ fully ground $g$ of the $g g$. More formally, I will write " $f f<_{a r b} g(g g)$ ". Here " $g(g g)$ " is to be understood such that $g$ is a fact (i.e. an obtaining state of affairs) and the $g g$ are a plurality of states of affairs, the choice plurality, such that $g$ is among them.

Regarding the connection between arbitrary grounding and metaphysical modality, it seems reasonable to deny that if $f f$ fully and arbitrarily ground $g$ of the $g g$, then necessarily, if all among the $f f$ obtain, then so does $g$ (given that $g g$ is a non-singular plurality). After all, $g$ is an arbitrarily chosen state of affairs from the $g g$ and it seems definitive of arbitrarily choosing one of the $g g$ that any other of the $g g$ could also have been chosen. Consequently, those who accept that there are cases of arbitrary grounding also have to accept that some facts are fully grounded without

\footnotetext{
1 I will take facts to be those states of affairs that obtain. Consequently, all facts are states of affairs, but not all states of affairs are facts. I will use " $f, g, \ldots$ " to refer to single states of affairs and " $f f, g g, \ldots$, to refer to pluralities of states of affairs. Every plurality consists of at least one element. This means that singular pluralities will be accepted as a limiting case.
} 
being necessitated by their grounds. I will not consider very general arguments against necessitating full grounds in this paper, but rather focus on the theoretical usefulness of arbitrary grounding. Of course, proponents of arbitrary grounding can still hold that non-arbitrary full grounding is necessary in the sense that the obtaining of the grounds necessitates the obtaining of the grounded. If they do so, they will also wish to accept that if the $f f$ fully ground $g$ of the $g g$, then necessarily, if all among the $f f$ obtain, then so does one of the $g .^{2}$

Every example that will be considered in what follows will be such that exactly one of those among the choice plurality obtains. This will be due to the candidate groundees being incompatible. In principle, however, I do not see any reason to not acknowledge cases of arbitrary grounding where it is also arbitrary how many of the states of affairs in the choice plurality obtain. Therefore I am reluctant to presuppose any general principles about these matters.

Moving away from toy-examples, I will present three metaphysical hypotheses that are best formulated in terms of arbitrary grounding in the next section. In Sect. 3 I will discuss how arbitrary grounding relates to non-arbitrary grounding and how it connects to the popular view that grounding is tightly connected to explanatory arguments or metaphysical laws. In Sect. 4 I will relate my proposal to extant proposals in the literature, namely the idea of stochastic grounding put forward by Bader (2021) and the idea that there are partial grounds without a corresponding full ground presented by Leuenberger (2020) and by Trogdon and Witmer (2021).

\section{Applications}

In this section, I try to show how doing grounding-based metaphysics can profit from having arbitrary grounding in the metaphysical tool-kit. I will present three independent metaphysical hypotheses from diverse areas of metaphysics that can be better understood and look more plausible when cast in terms of arbitrary grounding.

The first hypothesis, arbitrary reference, has been formulated independent of the grounding-literature by Breckenridge and Magidor (2012). I will show that the most plausible formulation of the doctrine of arbitrary reference in terms of grounding makes use of the notion of arbitrary grounding.

The second hypothesis consists in the claim that tensed facts (like the fact that Berta is sitting now) are grounded in non-tensed facts (like the fact that Anna is sitting on the 10th of June 2020 at $3 \mathrm{pm}$ ). I will argue that, given that the notion of obtaining is absolute and that reality is coherent, defending this hypothesis requires the employment of arbitrary grounding.

The third hypothesis combines the thesis that all non-qualitative facts are grounded in qualitative facts with the thesis that haecceitistic switches are possible.

\footnotetext{
${ }^{2}$ See Trogdon (2013) for arguments in favour and Skiles (2015) for arguments against non-arbitrary grounding being necessary in the described sense.
} 
I will show that making use of arbitrary grounding allows to maintain this combination of views and that this allows to meet some problems for groundingbased formulations of qualitativism. ${ }^{3}$

What all these applications have in common is that they motivate giving up on the idea that the fundamental modally fixes the non-fundamental. In all applications we will find scenarios in which it seems plausible to uphold that (i) there are different possible ways for the world to be that are compatible with the same base of fundamental facts and (ii) there is no explanation for which of these ways for the world to be obtains.

In what follows, I will make precise what it means for the fundamental to modally fix the non-fundamental. Let us say that worlds $w$ and $v$ are factindiscernible iff for every state of affairs $s$ : $s$ obtains in $w$ iff $s$ obtains in $v$. Let us further say that worlds $w$ and $v$ are fundamentality-indiscernible iff for every state of affairs $s$ : $s$ obtains and is ungrounded in $w$ iff $s$ obtains and is ungrounded in $v$. This allows to formulate the following principle:

Fundamental Fixing Necessarily, if worlds $w$ and $v$ are fundamentalityindiscernible, then worlds $w$ and $v$ are fact-indiscernible.

Some might object to Fundamental Fixing on grounds of being sceptical about foundationalism, the thesis that there is a fundamental level of facts that ground all other facts. There is a way to retain the spirit of Fundamental FIXING without committing to foundationalism. Let the $b b$ be a $w$-base iff every fact in $w$ is either among the $b b$ or fully (arbitrarily or non-arbitrarily) grounded in some of the $b b$. Now we can formulate the following foundationalist-failure-friendly principle:

BASE FIXING Necessarily, if some $b b$ are both a $w$-base and a $v$-base, then worlds $w$ and $v$ are fact-indiscernible.

It should be clear that the question whether there is a failure of BASE FIXING is distinct from the question whether there are cases where full grounds do not necessitate what they ground. First (and as already mentioned above), every example that will be discussed in this paper is compatible with non-arbitrary grounds necessitating what they ground. Second, putative failures of non-arbitrary grounds necessitating what they ground are compatible with BASE FIXING. Both Leuenberger (2014) and Skiles (2015) explicitly hold that their cases of contingent grounding are compatible with principles akin to BASE FIXING (see Skiles 2015, §5.2 and Leuenberger 2014, §V).

Although all of the hypotheses I will discuss in this section are controversial, discussing them will show how having the notion of arbitrary grounding at our disposal is helpful for metaphysical modelling. My aim in this paper is not do defend a particular case of arbitrary grounding. The claim I defend is that it is worthwhile to have arbitrary grounding in our metaphysical toolbox. I aim to show that we have to use arbitrary grounding to formulate some metaphysical hypotheses

\footnotetext{
${ }^{3}$ I take the application of stochastic grounding proposed in Bader (2021) to be another potential application for arbitrary grounding. I will discuss the relation between Bader's proposal and mine in Sect. 4.
} 
and some hypotheses that are best formulated in terms of arbitrary grounding deserve further consideration.

\subsection{Arbitrary reference}

The doctrine of arbitrary reference, which has been devised by Magidor and Breckenridge (Breckenridge \& Magidor, 2012), has it that the occurrence of " $x$ " in "Let $x$ be an arbitrary natural number between 1 and 4" refers to one of the four numbers $1,2,3,4$. It is epistemologically inaccessible which number it refers to and there are no non-semantic facts that settle to which number " $x$ " refers. Giving an account of sentences of this form is important because they frequently feature in mathematical proofs.

Arbitrary reference is not the only candidate account for providing a meaning for sentences like "Let $x$ be an arbitrary natural number." On some accounts the given occurrence of " $x$ " is taken to be an implicitly bound variable, on others the whole sentence is held to be a meaningless yet instrumentally useful device for reasoning. A competitor that accepts that " $x$ " is a referring expression is Kit Fine's account of arbitrary objects, which is based on the idea that the given occurrence of " $x$ " nonarbitrarily refers to a special kind of object, namely an arbitrary object (see e.g. Fine, 1985).

Arguing that arbitrary reference provides the best account of reasoning with the given kind of stipulation goes beyond the scope of this paper. ${ }^{4}$ This section will argue that acknowledging arbitrary grounding provides a place for arbitrary reference in the hierarchy of metaphysical priority. This gives the proponent of arbitrary reference a reason to accept arbitrary grounding. Furthermore, it shows how arbitrary grounding can be a useful tool for modelling metaphysical hypotheses.

Let us assume that " $x$ " in "Let $x$ be an arbitrary natural number" refers to the number 5. That this is the case seems to not be a fundamental fact, for it is a fact that involves such allegedly high-level phenomena as language, token-occurrences of sentences, and the semantic phenomenon of reference. For this reason, the proponent of arbitrary reference should accept that this fact is grounded. Clearly facts about language-use go some way to explain what " $x$ " in the given case refers to, they explain why $x$ refers to a natural number. At the same time it seems to be not only unexplained, but unexplainable why " $x$ " refers to the number 5 , rather than to the number 22. Non-semantic facts explain all there is to explain. Which of the natural numbers " $x$ " refers to is arbitrary.

The proponent of arbitrary grounding can accept that the fact that " $x$ " refers to the number 5 ([" $x$ " denotes 5]) is arbitrarily grounded with [" $x$ " denotes 1], [" $x$ " denotes 2],...being the choice-plurality. This perfectly captures the situation as the proponent of arbitrary reference sees it: There is an explanation for " $x$ " referring to the number 5, rather than to George Harrison. Still, there is no explanation

\footnotetext{
${ }^{4}$ See $\$ 2$ of Breckenridge and Magidor (2012) for arguments in favour of this conclusion and $\S 3$ of their paper for further potential applications of arbitrary reference.
} 
forthcoming for " $x$ " referring to the number 5 , rather than to the number 22 . Which natural number " $x$ " refers to is an arbitrary choice and it remains unexplained which element of the choice plurality obtains.

In what follows, I will argue that the use of arbitrary grounding allows to address a challenge that arises for the proponent of grounding-based metaphysics who wishes to make sense of arbitrary reference. Magidor and Breckenridge openly admit that it might seem implausible to many that semantic facts are not necessitated by non-semantic facts (see Breckenridge \& Magidor, 2012: p. 380). They take some solace in the arguments to the conclusion that the semantic does not supervene on the non-sematic provided in Kearns and Magidor (2012). However, the independent cases in which the semantic fails to supervene on the non-semantic discussed in Kearns and Magidor (2012) share certain features. In all these cases, a physicalistic world $w$ is compared with a physical duplicate of $w$ in which there are additional non-physical entities that account for a change in semantic facts. These comparisons are then used to argue that semantic facts are not necessitated by physical facts. In an additional step, it is argued that the failure of the physical facts to necessitate the semantic facts goes hand in hand with a failure of the nonsemantic facts to necessitate the semantic facts. Whatever the merits of these cases, upon closer examination it becomes clear that they are of a very different nature than what seems to go on in cases of arbitrary reference.

In particular, the examples in Kearns and Magidor (2012) are such that they respect the idea that there is no change in reference without a change in the fundamental. The physical duplicates of $w$ are such that some entities in these worlds are not brought about by anything we can find in $w$. The duplicates include some ungrounded facts that do not obtain at $w$. These are facts about the existence of non-physical ghosts or (in one of the examples) a very natural non-physical substance Kearns and Magidor call “ghost-water”. In each of Kearns and Magidor's cases, the worlds that semantically differ from a physicalistic world $w$ are such that they contain fundamental non-physical facts that do not obtain in $w$.

Is arbitrary reference plausible if one models it along the lines of the examples in Kearns and Magidor (2012)? Or do cases of arbitrary reference require failures of FundAMENTAL FIXING (or BASE FIXING in non-foundational worlds) to be plausible? If one wanted to retain Fundamental FiXING (or BASE FiXING) and allow for arbitrary reference, then one would have to claim that there is a difference in the fundamental (or in every base) between our world @ in which (so we might assume) " $x$ " in "Let $x$ be any natural number" refers to the number 5 and a physical duplicate of our world $w$ in which " $x$ " in the same occurrence of "Let $x$ be any natural number" refers to the number 22. What does this difference in the fundamental consists in? In at least one of @ and w, there would have to be a non-physical fundamental fact, a semantic decider, that is responsible for fixing the reference of " $x$ ". Given that one wishes to uphold that use-facts go some way to explaining the fact that " $x$ " refers to the number 5 , a story about how these semantic deciders could complement usefacts to ground reference-facts would have to be told. There seems to be no independently motivated account of such semantic deciders forthcoming and having to argue for them would presumably be unwelcome to the friend of arbitrary reference. This suggests that treating arbitrary reference as analogous to the 
examples in Kearns and Magidor (2012) is not promising. Arbitrary reference should be combined with an account of the explanation of reference-facts that allows for failures of BASE FIXING. The posit of arbitrary grounding fits the bill. It allows to make sense of failures of the non-semantic to necessitate the semantic without having to provide an account of semantic deciders or any other fundamental difference-makers.

I have argued that the cases presented in Kearns and Magidor (2012) do nothing to undermine BASE FIXING and that arbitrary reference is only plausible if BASE FIXING fails. This shows that the posit of arbitrary grounding should be welcome to the proponent of arbitrary reference, for arbitrary grounding allows to accommodate and account for failures of BASE FIXING. Arbitrary reference not only qualifies as a paradigmatic case of arbitrary grounding, it is also a metaphysical hypothesis that profits from the employment of arbitrary grounding. By using arbitrary grounding, the proponent of arbitrary reference can dodge the question which fundamental facts account for the difference between a world where our occurrence of " $x$ " refers to the number 5 and a world where it refers to the number 22 .

\subsection{Arbitrary tense}

In the debate about the metaphysics of time, it is customary to distinguish between tense-involving $A$-properties and non-tense-involving $B$-properties. Examples for $A$ properties are the property of sitting right now, the property of having been a child in the past, or the property (of a position in time) to be present. Examples for $B$ properties are the property of sitting on the 12th of June 2020, the property of being a child at some time that is earlier than the 12th of June 2020, or the property (of a position in time) to be later than the year 2020 .

Corresponding to the distinction between $A$-properties and $B$-properties, there is a distinction between $A$-theories of time and $B$-theories of time. How these theories are to be characterised is a matter of debate. It seems safe to say that proponents of $B$-theories either take there to be no $A$-facts (i.e. facts involving $A$-properties), or they take them less metaphysically seriously than the $B$-facts (i.e. facts involving only $B$-properties). Eliminativists who hold that there are no $A$-facts can still maintain that "Now is the year 2020" states a truth when uttered at time $t$ in the year 2020. However, they can only do so by holding that this sentence (and other sentences involving tense-vocabulary) states a $B$-fact like the fact [ $t$ is in the year 2020].

Can one be a $B$-theorist and still accept that there are facts involving $A$ properties? One way to accept that there are such facts and to take them less seriously than $B$-facts is to hold that all fundamental facts are $B$-facts. If one holds that all $A$-facts are grounded in fundamental $B$-facts, then one's theory plausibly qualifies as a $B$-theory (but see some discussion below). To not make the groundingformulation of the $B$-theory dependent on the assumption of foundationalism, it can be amended as follows:

Grounded $B$-Theory There are some $A$-facts and there is a base of the actual world that contains only $B$-facts. 
The $B$-theory is frequently formulated or characterised in terms of grounding in the present literature. ${ }^{5}$ Given that now is the year 2020, the proponent of GROUNDED $B$-THEORY will likely wish to ground the tensed fact [Now is the year 2020] in the base of $B$-facts, whereas they will hold that the tensed state of affairs [Now is the year 2030] does not obtain and hence is not grounded (by the factivity of grounding). In ten years, our proponent of GROUNDED $B$-THEORY might still not have changed their position on the matter. They will then hold that the very same $B$-facts that already obtained in the year 2020 have a ground of [Now is the year 2030] among them. The proponent of GROUNDED $B$-THEORY seems to be committed to the claim that it changes which states of affairs obtain.

How can the proponent of GROUNDED $B$-THEORY react to the seeming result that it changes across times which states of affairs obtain? There are two choice-points for the proponent of GROUNDED $B$-THEORY that correspond to the following two questions. The first question asks whether she takes the $A$-facts to obtain in a relativised and non-absolute way. The second question is directed at those who take obtaining to be absolute and asks them whether they accept that every $A$-state of affairs that has obtained, obtains or will obtain is such that it presently obtains simpliciter.

If the proponent of GROUNDED $B$-THEORY answers the first question affirmatively, then she might take the notion of obtaining to be relativised (or indexed) to points in time. If the sentence "Now is the year 2020" is uttered at a given point in time $t$ in the year 2020, then it says of the state of affairs [Now is the year 2020] that it obtains-at- $t$ (which is always and eternally true). If the same sentence is uttered at time $t^{\prime}$ in the year 2030, then it says of [Now is the year 2020] that it obtains-at- $t^{\prime}$ (which is always and eternally false). Generally, the sentence $\phi$ uttered at $t$ states that $[\phi]$ obtains-at- $t$. This allows the $B$-theorist to uphold that "Now is the year 2020" expresses an A-fact iff it is uttered in the year 2020 without having to maintain that it changes which states of affairs obtain at which points in time. ${ }^{6}$

If one answers the first question negatively, then one works with an absolute notion of obtaining. Those who answer it negatively face the second question. To reiterate, the second question asks whether the proponent of GROUNDED $B$-THEORY holds that every $A$-state of affairs that has obtained, obtains or will obtain does presently obtain simpliciter. The view that results from answering this second question affirmatively is committed to the result that some of the obtaining facts are incoherent. In the year 2020, the fact [Now is the year 2020] obtains. In the year

\footnotetext{
5 See e.g. Cusbert and Miller (2018) and Amijee (2021). My proposal in this section is related to and inspired by Amijee $(2021, \S 2.1)$, although the case is set-up differently and the consequences I draw differ from those drawn by Amijee.

6 This view corresponds to one way to understand temporalism with respect to propositions. E.g., Berit Brogaard takes temporalism to be the position that there is "genuinely propositional content [that] can be true or false only relative to a time" (Brogaard 2012). I should acknowledge that positing an obtaining-at$t$-relation might not be the only way to be a relativist (see e.g. Spencer, 2016 for discussion of alternative options). I will not discuss alternative options, for my aim in this section is not to provide arguments against relativism, but to show that there is an option to spell out the $B$-theory in terms of grounding without being a relativist or embracing incoherence.
} 
2030 the fact [Now is not the year 2020] will obtain. Allowing for such incoherent facts to both obtain simpliciter would amount to a variant of fragmentalism (see Fine, 2005; Lipman, 2015). The defender of GRounded B-THEORY could hold that the level of fundamental facts is coherent, whereas incoherence kicks in at a higher level. Coherent $B$-facts, so she might hold, are such that incoherent $A$-facts are grounded in them. According to this view it does not change which facts obtain, both [Now is the year 2020] and [Now is not the year 2020] always obtain, although they do so in different fragments of reality.

Answering any of the given questions affirmatively leads to an interesting position that deserves further consideration. I will not argue against any of the resulting positions, but I maintain that the option of answering both of the questions negatively deserves serious consideration. Maybe some variant of relativistic $B$ theory can be motivated, but it seems plausible that many $B$-theorists will wish to avoid embracing relativism. There is at least some motivation for holding that "Now is the year 2020" states a non-relativistic tensed fact when uttered in the year 2020.

Concerning the second question, I have to admit that I find the position that the world is fundamentally coherent albeit derivatively incoherent fascinating. Nevertheless, it seems clear that incoherence is a result that many $B$-theorists will wish to avoid. The question that arises is whether there is a variant of GRounded $B$-THEORY on which reality is coherent and there is only one absolute notion of obtaining.

Independent from the individual misgivings about answering any of the questions affirmatively, there is a positive reason to answer both of them negatively. Doing so would amount to accepting that it changes which facts obtain. It would allow to hold that if [Now is the year 2020] obtains, then this gives the year 2020 has a special metaphysical status, a status which the year 2030 lacks. In ten years, it will be the case that the year 2030 has acquired the special status of being present which is now held by the year 2020. That the present point in time does have this special metaphysical status is the intuition that drives many into the arms of $A$-theorist. ${ }^{7}$ If the $B$-theorist could accommodate that (i) [Now is the year 2020] obtains simpliciter and (ii) it is not the case that [Now is the year 2030] obtains simpliciter, then she found a way to accommodate this intuition that motivates many $A$-theorists.

Some might say that acknowledging that the year 2020 has such a special metaphysical status amounts to adopting $A$-theory in disguise and should be avoided by the $B$-theorist. However, there is a lot of intuitive support for the thesis that (at the time this paper is written) the year 2020 is metaphysically privileged. Therefore it is at least worth exploring whether one can uphold it together with GROUNDED $B$ THEORY. Maybe there is room for disagreement about nomenclature: Should the view qualify as properly $B$-theoretic? Maybe it is best thought of as some kind of hybrid view. But whatever one's verdict when it comes to labelling, the view has some initial attraction. Combining the view that all fundamental facts are $B$-facts

\footnotetext{
7 See e.g. Zimmerman's remark that an affirmative answer to the question "Are present events and things somehow more 'real' than those wholly in the past or future?" sounds "obvious and commonsensical, at least to me" (2007).
} 
with the view that there are changing $A$-facts promises to account for $A$-theoretic intuitions while maintaining the priority of the $B$-series.

If one wishes to spell out this view, one is confronted with the following problem. Let the $b b$ be our base of $B$-facts. Now it is the case that the $b b$ are a base and [Now is the year 2020] obtains. It will be the case that the $b b$ are a base and [Now is the year 2030] obtains. One might wish to uphold that whatever will be the case is metaphysically possible. This commits the proponent of the view under discussion to a failure of BASE FIXING. The dialectical situation is akin to the one discussed in the last subsection. There is a view which presents us with a metaphysical explanation that fails to discriminate between a number of candidate groundees. Again, taking recourse to arbitrary grounding will allow to formulate the case under discussion.

There is a complication that is due to the $A$-facts being systematically coordinated. If the year 2020 is present, then the year 1900 is past and the year 2030 is future. This suggests that the tensed facts are grounded en bloc. To make grounding en bloc intelligible, one has to combine arbitrary grounding with plural grounding. Plural grounding that is not reducible to multiple cases of singular grounding has been introduced to the debate by Dasgupta (2014). The underlying idea is that in a case of plural grounding, the grounds bring about a plurality of facts that is grounded at once. Combining plural grounding with arbitrary grounding requires employing the notion of a superplurality. A superplurality fff relates to a plurality $f f$ as second order plural quantification relates to first order plural quantification. For the present purpose, we might understand a superplurality as a plurality of pluralities. However, this way to understand it seems to wrongly presuppose that pluralities are entities and should hence be treated with care. ${ }^{8}$ With the notion of a superplurality in place, one can say that the $f f$ ground the $g g$ of the ggg.

Call all the plurality of $A$-facts that obtain (right now) the $A$-series. The proposed view is that the base of $B$-facts plurally grounds the $A$-series. The other pluralities in the choice-superplurality are the other candidates for the $A$-series that are compatible with the given $B$-series and are grounded in it at earlier or later times.

Discussing the merits and potential problems for the view that the $A$-series is arbitrarily grounded in the base of $B$-facts goes beyond the scope of this paper. One interesting question for further research is whether the described position has more or less problems than standard variants of the $A$-theory to account for the passage of time. I would not be surprised if there were some insurmountable obstacles to defending the view, but it seems clear that a view that allows combining the claims that only $B$-facts are fundamental and that the present is metaphysically special deserves some attention. Arbitrary grounding is needed to formulate this view. Therefore, if we should discuss the view, we should make use of arbitrary grounding as a tool for theoretical modelling.

\footnotetext{
${ }^{8}$ See Rayo (2006) for a thorough discussion of superplural quantification and superpluralities.
} 


\subsection{Arbitrary haecceities}

This subsection discusses the relation between the following two theses:

Haecceitism Possibly, the following two conditions hold for some $q q$ and $r r$ :

- The $q q$ are all and only the qualitative facts and the $r r$ are all and only the non-qualitative facts

- It is possible that the $q q$ are all and only the qualitative facts and the $r r$ are not the plurality of all and only the non-qualitative facts.

Qualitative Base Necessarily, there is a base of qualitative facts $b b$ such that every non-qualitative fact is fully grounded in some facts among the $b b$.

For foundationalists, Qualitative BASE is tantamount to the thesis that necessarily, the fundamental facts ground all other facts and all fundamental facts are qualitative. HAECCEITISM can be captured by the slogan that possibly the world possibly differs with respect to non-qualitative facts without differing with respect to qualitative facts. I will start by clarifying these two theses and then turn to briefly motivating their acceptance. Then I will show that they turn out to be incompatible with BASE FIXING and can be reconciled by employing arbitrary grounding.

In my statements of both HaEcceitism and Qualitative Base, I have made use of the notion of a qualitative fact. What is it for a fact to be qualitative? Although it is disputed how and whether qualitativeness can be defined, an informal elucidation will do for the present purpose: ${ }^{9}$ Qualitative properties are those properties that don't involve any individuals. Redness is a paradigm example of a qualitative property, whereas being married to Donald Trump is not. Analogously, one might define qualitative facts as facts that don't involve any individuals or non-qualitative properties. The fact of there being a red circle is an example of a plausibly qualitative fact.

The idea standing in the background of Qualitative BASE is that all there fundamentally is to reality concerns which kinds of objects there are and what they are qualitatively like. Here are some reasons friends of QuALITIATIVE BASE and related theses provide for subscribing to this idea: Fundamental non-qualitative facts would be impossible to find out. At the same time, they don't play any indispensable role in physical theories. Ontological parsimony suggests doing away with them and some hold that postulating fundamental facts that are epistemologically hidden from us should be avoided. This motivation is clearly defeasible, but it shows that if QuAlitiative BASE is a coherent hypothesis, then it should be taken seriously. ${ }^{10}$

I will follow Dasgupta (2014) in holding that the grounding of the non-qualitative by the qualitative should be regimented in terms of plural grounding. One of Dasgupta's reasons for doing so, which I mention because it will be relevant for my

\footnotetext{
9 See e.g. Hoffmann-Kolss (2019) and Cowling (2015) for discussion on how and whether to define qualitativeness.

${ }^{10}$ For recent defences of the thesis that all fundamental facts are qualitative see Paul (2012) and Dasgupta (2009).
} 
argument below, is that it allows to give plausible explanations for non-qualitative facts in a Max-Black-world in which there is nothing but two indistinguishable spheres $A$ and $B$. The problem for the non-pluralist is that she presumably has to find qualitative facts that explain the existence of $A$ without explaining the existence of $B$. Facts that non-plurally explain the existence of $A$ as well as the existence of $B$ seem to be too unspecific to their respective explanatory targets. However, given the qualitative indistinguishability of $A$ from $B$, it seems impossible to come up with any qualitative facts that ground $A$ without grounding $B$. The defender of plural grounding has the promising option to provide a ground for the plurality $A, B$ that grounds both existence-facts collectively (see Dasgupta, 2014: p. 25f).

HaEcCeitism is the modal thesis that denies that qualitative facts necessitate all non-qualitiatve facts. Here is a reason to subscribe to HAECCEITISM if one allows for a Max-Black-world with two indistinguishable spheres $A$ and $B$ : From the perspective of this world, it seems possible that $A$ exists on its own and that $B$ exists on its own. These are two distinct possibilities. However, the two possibilities are qualitatively indistinguishable. A related case can be constructed if we assume that in our MaxBlack-world, at some point in time $t, A$ ceases to exist and $B$ stays alone from then on. It seems also be possible from the perspective of this world that the other sphere, $B$, ceases to exist at $t$ and $A$ stays alone from then on. Again, the possible worlds witnessing these distinct possibilities are qualitatively indistinguishable. ${ }^{11}$

Upholding both Qualitative BASE and HaEcCEITISM requires a failure of BASE Fixing. Qualitative Base has it that at every possible world $w$, there there are some qualitative $b b$ that form a $w$-base. Combined with HAECCEITISM, this yields that for some possible worlds $v$ and $w$ that are not fact-indiscernible, some qualitative $b b$ are both a $v$-base and a $w$ base. BASE FIXING says that this is not the case.

For this reason, BASE FIXING has to give way if we want to combine QuAlitative BASE and HaECCEITISM. As we have seen in the previous two subsections, arbitrary grounding allows for failures of BASE FIXING.

That the $f f$ arbitrarily ground $g$ of the $g g$ does not yield the result that the $f f$ collectively necessitate $g$. Some other of the $g g$ could have obtained instead of $g$.

This allows to uphold that from the perspective of a Max-Black-world, it is possible that $A$ is alone and possible that $B$ is alone. Let $g_{A}$ be the fact that $A$ exists and $g_{B}$ be the fact that $B$ exists. One might now hold that at one world some qualitative facts $f f$ arbitrarily ground $g_{A}$ of $g_{A}, g_{B}$ and at another world the same facts $f f$ arbitrarily ground $g_{B}$ of $g_{A}, g_{B} \cdot{ }^{12}$

This shows how acknowledging arbitrary grounding can reconcile qualitativism with haecceitism. Qualitativism says that every two qualitatively indistinguishable possible ways for the world to be share a grounding-base. Haecceitism says that some qualitatively indistinguishable possible ways for the world to be are different. Arbitrary grounding reconciles the two by providing a framework that allows to hold that different possible ways for the world to be share a grounding base.

\footnotetext{
11 For very similar examples see Russell (2017).

12 Presumably there are more states of affairs in the choice plurality, for one might also wish to allow for worlds with three, four or infinitely many indistinguishable spheres that are also indistinguishable from the spheres in the Max-Black-world.
} 
This discussion is not only relevant to the defenders of qualitativism. The question of how to ground non-qualitative facts about non-fundamental individuals is also pressing for those who do not subscribe to the hypothesis that there is a qualitative base. Even if you allow for fundamental non-qualitative facts about individual atoms, say, there might be problems about grounding the non-qualitative facts about the things that are made of these atoms.

Taking up an example presented in Skiles (2015), one might ask what grounds that Sally the sandwich exists. It seems plausible to hold that atoms $a_{1}, a_{2}, \ldots$ being arranged sandwich-wise is a plausible candidate. Using the case of a series of shipof-Theseus-style-operations on the sandwich, Skiles argues that possibly, $a_{1}, a_{2}, \ldots$ are arranged in the very same way and Sally does not exist. In such a scenario, a different sandwich is made of $a_{1}, a_{2}, \ldots$. For reasons of space, I will not further discuss potential reactions to examples of this type. ${ }^{13}$ However, the example indicates that the question how to ground non-qualitative facts about higher-level entities in facts about fundamental (or lower-level) entities also arises for those who allow that there are non-qualitative facts.

Arbitrary grounding allows to maintain that e.g. $a_{1}, a_{2}, \ldots$ ground Sally in some worlds and Salomon (another sandwich) in other worlds. More generally, it allows to hold that facts about low-level entities ground facts about high-level entities without modally fixing them. This suggests that every one who worries about the grounds of the non-qualitative facts concerning particular houses, sandwiches, or planets should take interest in the options arbitrary grounding offers them.

\section{Connections}

Some readers might find it off-putting that postulating arbitrary grounding seems to add (yet) another variant of grounding to the metaphysical picture. Worries of this sort can be mitigated by showing that arbitrary grounding is not an unconnected posit, but smoothly integrates with non-arbitrary grounding. In this section, I show that non-arbitrary grounding can be defined as a limiting case of arbitrary grounding and I discuss the claim that arbitrary grounding can be defined in terms of nonarbitrary grounding. I will furthermore argue that arbitrary grounding naturally integrates into a view on which grounding is tightly connected to explanatory arguments and metaphysical laws.

I claim that non-arbitrary grounding can be conceived of as a limiting case of arbitrary grounding. A case of non-arbitrary full grounding only differs from a case of proper arbitrary grounding insofar as the choice-plurality has exactly one state of affairs among it, namely the fully grounded fact. There is no way to non-trivially choose from such a plurality, that $g$ is chosen from the plurality that has only $g$ among it is guaranteed.

Treating non-arbitrary grounding as a limiting case of arbitrary grounding allows us to define non-arbitrary grounding in terms of arbitrary grounding:

\footnotetext{
13 See Skiles (2015) $\$ 3$ for a detailed discussion.
} 
- The $f f$ fully (non-arbitrarily) ground $g$ iff the $f f$ fully arbitrarily ground $g$ of $g$. (Formally: $f f<g$ iff $f f<_{a r b} g(g)$ )

The situation is similar to the introduction of plural grounding. When introducing plural grounding into the picture, cases of grounding where a plurality nondistributively grounds a further plurality, this could be done without having to countenance two distinct kinds of grounding. Singular grounding could be recaptured in terms of plural grounding, namely as a case where the grounded plurality is a singular fact.

Some might be tempted to claim that arbitrary grounding can be defined in terms of non-arbitrary grounding. Letting $\bigvee g g$ be the disjunctive state of affairs that at least one of the $g g$ obtains, the following definition might be proposed: ${ }^{14}$

- The $f f$ fully arbitrarily ground $g$ of the $g g$ iff

- The $f f$ fully non-arbitrarily ground $\bigvee g g$

- No $g^{\prime}$ among the $g g$ and $f f^{\prime}$ among the $f f$ are such that the $f f^{\prime}$ fully ground $g^{\prime}$

- $g$ obtains

However, there might be cases in which the definiendum is fulfilled, but that nevertheless do not plausibly qualify as cases of arbitrary grounding. Such a case would arise if one accepted the following two principles:

Essence Grounds Prejacents For all $x x$ : If $\square_{x x} \phi$, then $\left[\square_{x x} \phi\right]$ fully grounds $[\phi]$ Essential LEM For some $\quad x x: \quad \square_{x x}(\phi \vee \neg \phi) \quad$ and $\left(\neg \square_{x x} \phi\right) \wedge\left(\neg \square_{x x} \neg \phi\right)$

Assume that (i) Essence Grounds Prejacents (whose name I borrow from Zylstra 2019) holds, that (ii) it is jointly essential to the operations of disjunction and negation (maybe together with the objectual content of $\phi$ ) that $(\phi \vee \neg \phi)$, and that (iii) $\phi$ obtains. Now the above definition yields that the mentioned essentialist fact would arbitrarily ground $[\phi]$ of $[\phi],[\neg \phi]$. However, it doesn't seem to be the case that the essentialist fact gives rise to an arbitrary choice and thereby arbitrarily grounds $[\phi]$. After all, the schematic principles are supposed to hold for all instances of " $\phi$ ". If they would give rise to arbitrary grounding, one would get the result that every fact is arbitrarily grounded.

Of course, Essence Grounds Prejacents and Essential LEM are controversial priniciples. One way to argue against the combination of EssENCE GROUNDS Prejacents and Essential LEM is to point out that it violates the seemingly plausible rule that every full ground of a disjunctive fact $[\phi \vee \psi]$ has one of $[\phi],[\psi]$ or a full ground of one of $[\phi],[\psi]$ among it. This rule has been proposed by Fine, who informally describes it as follows: "[w]hat we would like to say is that the grounds for a disjunction (...) should be mediated through its disjuncts" (Fine, 2012: p. 64). ${ }^{15}$

\footnotetext{
14 Thanks to Alexander Skiles for proposing a similar definition.

15 A similar argument against Essence Grounds Prejacents has been proposed in Glazier (2017). For another argument against Essence Grounds Prejacents see Zylstra (2019). See Vogt (2021) for a response to Zylstra.
} 
However, insisting on this mediation-rule is not a promising strategy for the defender of the above definition of arbitrary grounding: The mediation-rule precludes that the definiendum is ever fulfilled, for the definition says that cases of arbitrary grounding are cases of grounding in which the ground of a disjunction is not mediated through any of its disjuncts. Therefore, those friends of arbitrary grounding who accept the mediation rule cannot accept the above definition. ${ }^{16}$

It should be noted that the tension with the mediation-rule does not arise if one accepts primitive arbitrary grounding without defining it in terms of non-arbitrary grounding. If $g g$ is the choice-plurality of a case of arbitrary grounding, then one might still hold that the grounds of $\bigvee g g$ have to be mediated through one of the $g g$, although it is arbitrary through which one they are mediated. Neither does the tension with Essence Grounds Prejacents and Essential LEM arise, for if arbitrary grounding is a primitive notion, then one can accept both these principles without having to hold that they give rise to a case of arbitrary grounding.

For these reasons, I do not wish to commit to the above definition of arbitrary grounding. However, I am also not committed to its not being adequate. If the worries I mentioned can be defused, then the definition might well work. For the time being, however, I propose to accept arbitrary grounding as primitive.

Further support for the thesis that arbitrary grounding is an acceptable primitive posit comes from comparing it to multiple conclusion logic. In multiple conclusion logic, some of the deduction rules don't allow you to deduce a single conclusion, but rather allow you to deduce that some of multiple conclusions are true. A simple and intuitive example of such a rule allows to deduce that some of $\phi, \psi$ are true from $\phi \vee \psi{ }^{17}$

This rule of disjunction-elimination also shows why one should be inclined to think that there is room for a notion of irreducible multiple conclusion logic. One might be tempted to say that the claim that $\phi_{1}, \phi_{2}, \ldots$ allows to deduce (some of) $\psi_{1}, \psi_{2}, \ldots$ in multiple-conclusion logic reduces to the claim that $\phi_{1}, \phi_{2}, \ldots$ allows to deduce $\psi_{1} \vee \psi_{2} \vee \ldots$ in ordinary single-conclusion logic. But this would yield the verdict that the multiple-conclusion-rule of disjunction-elimination $\phi \vee \psi \vdash_{M C L}$ $\phi, \psi$ reduces to an instant of the rule of self-entailment of the form $\phi \vee \psi \vdash_{S C L} \phi \vee \psi$, which runs counter to the demand that the rule of disjunctionelimination says something informative about the nature of disjunction.

There is an analogy between multiple-conclusion logic (and its relation to singleconclusion logic) and arbitrary grounding (and its relation to non-arbitrary grounding). In this analogy, the analogue to rules of deduction (in the case of logic) are rules of ground (in the case of grounding). The analogy is particularly pertinent if one models grounding with valid explanatory arguments. Litland

\footnotetext{
${ }^{16}$ One might try to reformulate the definition in terms of a ground for the existentially quantified fact that some of the $g g$ obtains that has not a ground for any one of the $g g$ among it. This would, however, not structurally change the given dialectics. EssENTIAL LEM could be replaced by the claim that it is jointly essential to existential quantification and negation (and the objectual content of $\phi$ ) that some of $[\phi],[\neg \phi]$ obtains and a mediation-rule for existentially quantified facts seems to be equally plausible as the mediation-rule for disjunctive facts.

17 See e.g. Shoesmith (1978) for an introduction to multiple-conclusion logic.
} 
(2015, 2017) has suggested that a subclass of arguments that are valid in a given calculus are explanatory arguments. Explanatory arguments have their status in virtue of being based on rules of inference that have the status of being explanatory. To give examples, arguably the rule of disjunction introduction is explanatory (and hence $[\phi]$ grounds $[\phi \vee \psi]$, given that $[\phi]$ obtains) and the rule of conjunctionelimination is not (and hence the truth of $\phi \wedge \psi$ allows to infer the truth of $\phi$, but this inference-rule does not give us that $[\phi \wedge \psi]$ grounds $[\phi]$ ). Litland suggests that every case of grounding can be modelled by chaining such explanatory arguments. Those who wish to follow Litland in modelling grounding in this way can make sense of arbitrary grounding by holding that the underlying logic allows for multiple-conclusion inferences and that some of these multiple-conclusion inferences are explanatory.

A similar story can be told if one presupposes a metaphysical picture where cases of grounding are underwritten by laws of metaphysics (conceived of in analogy to laws of nature). This idea has recently gained support (see e.g. Rosen, 2017) and can also be spelled out in a way according to which grounding is connected to deductive arguments in which productive laws of metaphysics figure as premises (see e.g. Wilsch, 2016). If one allows that some such productive laws are such that they say that some facts produce one of a number of outcomes without specifying which one, then these laws can plausibly underwrite cases of arbitrary grounding.

\section{Comparisons}

This section discusses the relation between arbitrary grounding and related proposals that can be found in the recent literature. The first subsection will relate my proposal to stochastic grounding as it has been put forward by Bader (2021). In the second subsection I will discuss partial grounds without a corresponding full ground as proposed by Leuenberger (2020) and in Trogdon and Witmer (2021).

\subsection{Arbitrary grounding and stochastic grounding}

In a recent article, Ralf Bader proposed the idea that there might be cases in which there "are grounds that favour incompatible outcomes" (Bader, 2021). If, according to Bader's proposal, the $f f$ and the $g g$ are such opposing grounds (or groundcandidates), then there are incompatible groundees $h_{F}$ and $h_{G}$ favoured by the respective grounds. Bader holds that in these cases it is "up to chance which ground wins out" (Bader, 2021).

This proposal is elegantly tailored to the case Bader wishes to address with the employment of stochastic grounding. The problem he addresses is that "in a symmetric fission case, each fission-product $B$ and $C$ has an equal claim to being identical to the person $A$ that underwent fission" (Bader, 2021). In such a case it is reasonable to say that facts concerning the way $B$ relates to $A$ speak in favour of $B$ being identical to $A$, whereas facts concerning the way $C$ relates to $A$ speak in favour of the incompatible outcome that $C$ is identical to $A$. 
Arbitrary grounding (as it is proposed in this paper) has a broader range of applications than Bader's stochastic grounding. In particular, there are two features of Bader's proposal that limit its potential use. The first feature is that it builds the idea of a chancy element into the framework. I have two reservations about this. I do not see any good arguments for presupposing that every non-determined case of grounding has to be chancy. If taken metaphysically seriously, chance-distributions are a way to bring order into what is metaphysically not determined. Maybe one should resist the temptation to bring order and accept that some cases of metaphysical indeterminacy are simply arbitrary, or brute, and there is nothing more substantial one can say. ${ }^{18}$ The second reservation is of a more technical nature. When dealing with choice-pluralities of higher cardinalities, it is unclear whether one can coherently ascribe chances to the individual possible outcomes. Although there are ways to model lotteries on countably infinite sets like the natural numbers (see e.g. Wenmackers \& Horsten, 2013), it seems that e.g. the doctrine of arbitrary reference could be applied for number-systems of arbitrary cardinality. One might even be tempted to think that we can arbitrarily refer to some surreal number, although the surreal numbers are proper class many (see e.g. Conway, 2000 on surreal numbers). ${ }^{19}$

The second potentially troubling feature of Bader's proposal is that it can only be applied if there are opposing grounds (or ground-candidates). However, there might be cases in which this is not the case. I have proposed two potential applications that cannot be plausibly reconstructed in terms of opposing grounds: The hypothesis of arbitrarily grounded tense (Sect. 2.2) and the hypothesis of arbitrarily grounded haecceities (Sect. 2.3). For example, in the case of tensed facts I suggested that all $B$-facts together plurally ground all the $A$-facts, but there are incompatible combinations of $A$-facts that are possibly grounded by the very same $B$-facts. Even if one remains unconvinced by my particular proposals, I think they serve to show that there is room for non-determining grounding without opposing grounds.

Does Bader's proposal do a better job than mine when it comes to opposing grounds? If the $f f$ and the $g g$ are opposing grounds for $h_{f f}$ and $h_{g g}$, respectively, should one follow him in saying that the winning grounds alone ground the winning groundee? It seems that if $h_{f f}$ obtains, then it does so because the $f f$ and the $g g$ together fail to determine a clear winner and $h_{f f}$ is the lucky ground chosen from $h_{f f}$ and $h_{g g}$. This complex situation is ignored by the claim that the ff fully ground $h_{f f}$. The claim that $f f$ and $g g$ together arbitrarily ground $h_{f f}$ of $h_{f f}, h_{g g}$ seems to better capture the situation. In particular, my proposal allows for a more fine-grained view

\footnotetext{
18 One could take chance-ascriptions to be non-substantial. For example, one could say that if there is nothing in reality that distinguishes between two outcomes and we have no information about which of them obtains, then we should ascribe equal probability to each outcome. In this case, so it seems, chanceascriptions should not be part of the metaphysical story of arbitrary grounding, but rather of a story about how we should rationally deal with the arbitrary.

19 This would require there being a choice-plurality that has proper class many facts among it. I admit that one might have motivated reasons to deny this. However, it should also be noticed that the ideology of plural quantification (which is tightly connected to talk about pluralities) has, among other applications, been introduced to make sense of claims like "There are some things that are too many to form a set" (see e.g. Linnebo, 2014, §4.3).
} 
on the modal consequences of countenancing non-determining grounds. I can distinguish the question whether there are cases of arbitrary grounding from the question whether full non-arbitrary grounding is necessitating. For this reason, I am not concerned about my proposal not yielding the result that it is the winning ground alone that does the grounding work.

Finally, it should be noted that nothing prevents me from allowing that in some cases of arbitrary grounding, the grounds in fact do ground a particular chancedistribution. Of course, for this an account of such a chance-distribution would have to be provided. But if this can be done (and it has to be done to make sense of Bader's proposal, given that he takes chance-distributions seriously), then chancedistributions can freely be added to the present account.

\subsection{Arbitrary grounding and weak fundamentality}

Leuenberger (2020) has argued in favour of countenancing cases of partial grounding without corresponding cases of full grounding. Further cases of this sort have been proposed in Trogdon and Witmer (2021). Partially grounded facts that are not fully grounded are called "weakly fundamental" by Leuenberger. What is the exact relation between weak fundamentality and arbitrary grounding?

Whereas weakly fundamental facts do not have a full ground, I claim that a full arbitrary ground is a full ground. An arbitrary full ground of a particular fact is full in the sense that it is sufficient for the obtaining of one of the facts in the choice plurality. It fully explains that one of some facts obtains, although it does not explain which one. The sense in which this amounts to a full explanation becomes clear when one looks at the proposed applications. In the candidate-cases of arbitrary grounding considered in this paper, the candidate-groundees in the choiceplurality are all facts of a particular kind (semantic facts, A-facts, and hacceitistic facts, respectively), whereas the grounds are facts of another kind (non-semantic facts, B-facts, and qualitative facts, respectively). Given that the proposed cases of arbitrary grounding hold, there being semantic facts in a world that is fundamentally non-semantic is fully explained, and so is the obtaining of tensed facts in a world that has a non-tensed base and the obtaining of non-qualitative facts in worlds with a purely qualitative base.

This is an important feature that distinguishes arbitrary grounding from many putative cases of weak fundamentality. To show this, I present a putative case of weak fundamentality that clearly does not qualify as a case of arbitrary grounding. The case is taken from a paper by Trogdon and Witmer (2021) who attribute it to Kevin Mulligan: "Consider a simple atomic fact—some object $a$ has property $F$. Suppose that this fact lacks full grounds, as $a$ and $F$ do not decompose into more basic objects and properties. There is some plausibility to the claim that this fact is nevertheless partially grounded by the fact that $a$ exists." (Trogdon \& Witmer, 2021: p. 5) If one were to accept that $[a$ is $F]$ is partially grounded in [ $a$ exists] and that there is no full ground of $[a$ is $F]$, then the example would qualify as an example as a case of weak fundamentality. However, it would be implausible to claim that it also constitutes an example of arbitrary grounding. 
But does this show that being arbitrarily grounded is sufficient for not being weakly fundamental? If the $f f$ arbitrarily ground $g$ of the $g g$, then there is something unexplained about $g$ (namely why $g$ rather than another one of the $g g$ obtains). Some might take this to be sufficient for $g$ being weakly fundamental.

I believe that more research would be needed to settle this question and to make clear what exactly is at issue when the question whether arbitrary grounding leads to weak fundamentality is discussed. Carrying out this research is a task that has to wait for another occasion. ${ }^{20}$

Whatever the final verdict about the relation between weak fundamentality and arbitrary grounding might be, it is clear that arbitrary grounding merits a discussion in its own right. Even if being arbitrarily grounded is a way for a fact to be weakly fundamental, it still is a very specific way to be weakly fundamental, one that has its own class of promising applications and that is distinct from other candidates for weak fundamentality (like the one concerning the existence of an object $a$ being a partial ground of the weakly fundamental fact that $a$ is $F$ ). If being fully arbitrarily grounded is a way to be weakly fundamental, it is a way to be weakly fundamental that is independently interesting and deserves being investigated in its own right.

Acknowledgements Audiences at the Metaphysical Explanation III-conference in Lund and at the University of Bern asked challenging and helpful questions. Alexander Skiles provided me with an excellent comment at the conference in Lund. I had a very fruitful discussion about a previous draft with Gaetan Bovey, Hagen Braun, Martin Glazier, Henrik Rydéhn, Lisa Vogt, Michael Wallner, and Tobias Wilsch during a session of our impromptu reading group in the spring and summer of 2020. An anonymous reviewer for Philosophical Studies helped me to significantly improve this paper. I am very grateful to all these people.

Funding Open Access funding provided by Universität Bern.

Open Access This article is licensed under a Creative Commons Attribution 4.0 International License, which permits use, sharing, adaptation, distribution and reproduction in any medium or format, as long as you give appropriate credit to the original author(s) and the source, provide a link to the Creative Commons licence, and indicate if changes were made. The images or other third party material in this article are included in the article's Creative Commons licence, unless indicated otherwise in a credit line to the material. If material is not included in the article's Creative Commons licence and your intended use is not permitted by statutory regulation or exceeds the permitted use, you will need to obtain permission directly from the copyright holder. To view a copy of this licence, visit http:// creativecommons.org/licenses/by/4.0/.

\section{References}

Amijee, F. (2021). Explaining contingent facts. Philosophical Studies, 178, 1163-1181. https://doi.org/ 10.1007/s11098-020-01487-y.

\footnotetext{
${ }^{20}$ One potential question for further research concerns the modal profile of nothing over and above-ness. The definition of full grounding suggested in Trogdon and Witmer (2021) has it that some facts ff can only count as a full ground of a further fact $g$ if $g$ is nothing over and above the $f f$. If this proposal were adopted, the question whether a fact can be nothing over and above some further facts that do not necessitate it would become pertinent.
} 
Bader, R. (2021). The fundamental and the brute. Philosophical Studies, 178, 1121-1142. https://doi.org/ 10.1007/s11098-020-01486-z.

Breckenridge, W., \& Magidor, O. (2012). Arbitrary reference. Philosophical Studies, 158(3), 377-400. https://doi.org/10.1007/s11098-010-9676-z.

Brogaard, B. (2012). Transient truths: An essay in the metaphysics of propositions. Oxford University Press.

Conway, J. (2000). On numbers and games. Ak Peters Series. Taylor \& Francis.

Cowling, S. (2015). Non-qualitative properties. Erkenntnis, 80(2), 275-301.

Cusbert, J., \& Miller, K. (2018). The unique groundability of temporal facts. Philosophy and Phenomenological Research, 97(2), 410-432. https://doi.org/10.1111/phpr.12374.

Dasgupta, S. (2009). Individuals: An essay in revisionary metaphysics. Philosophical Studies, 145(1), 35-67.

Dasgupta, S. (2014). On the plurality of grounds. Philosophers' Imprint, 14(20).

Fine, K. (1985). Reasoning with arbitrary objects. Blackwell.

Fine, K. (2005). Tense and reality. In K. Fine (Ed.), Modality and tense (pp. 261-320). Oxford University Press.

Fine, K. (2012). Guide to ground. In F. Correia \& B. Schnieder (Eds.), Metaphysical grounding (pp. 37-80). Cambridge University Press.

Glazier, M. (2017). Essentialist explanation. Philosophical Studies, 174(11), 2871-2889.

Hoffmann-Kolss, V. (2019). Defining Qualitative Properties. Erkenntnis, 84, 995-1010. https://doi.org/ 10.1007/s10670-018-9991-x.

Kearns, S., \& Magidor, O. (2012). Semantic sovereignty. Philosophy and Phenomenological Research, 85(2), 322-350. https://doi.org/10.1111/j.1933-1592.2012.00624.x.

Leuenberger, S. (2014). Grounding and necessity. Inquiry, 57(2), 151-174.

Leuenberger, S. (2020). The fundamental: Ungrounded or all-grounding? Philosophical Studies, 177, 2647-2669. https://doi.org/10.1007/s11098-019-01332-x.

Linnebo, Ø. (2014). Plural quantification. In E. N. Zalta (Ed.), The stanford encyclopedia of philosophy, fall 2014 edn.

Lipman, M. A. (2015). On Fine's fragmentalism. Philosophical Studies, 172(12), 3119-3133. https://doi. org/10.1007/s11098-015-0460-y.

Litland, J. E. (2015). Grounding, explanation, and the limit of internality. Philosophical Review, 124(4), 481-532.

Litland, J. E. (2017). Grounding ground. Oxford Studies in Metaphysics, 10, 279-316.

Paul, L. A. (2012). Building the world from its fundamental constituents. Philosophical Studies, 158(2), 221-256.

Rayo, A. (2006). Beyond plurals. In A. Rayo \& G. Uzquiano (Eds.), Absolute Generality (pp. 220-54). Oxford University Press.

Rosen, G. (2017). Ground by law. Philosophical Issues, 27(1), 279-301.

Russell, J. S. (2017). Qualitative grounds. Philosophical Perspectives, 30(1), 309-348. https://doi.org/10. 1111 phpe.12078.

Shoesmith, D. J. (1978). Multiple-Conclusion Logic. Cambridge University Press.

Skiles, A. (2015). Against grounding necessitarianism. Erkenntnis, 80(4), 717-751.

Spencer, J. (2016). Relativity and degrees of relationality. Philosophy and Phenomenological Research, 92(2), 432-459. https://doi.org/10.1111/phpr.12153.

Trogdon, K. (2013). Grounding: Necessary or contingent? Pacific Philosophical Quarterly, 94(4), $465-485$.

Trogdon, K., \& Witmer, D. (2021). Full and Partial Grounding. Journal of the American Philosophical Association, 7(2), 252-271. https://doi.org/10.1017/apa.2020.26.

Vogt, L. (2021). Two problems for Justin Zylstra's truthmaker semantics for essence. Inquiry: An Interdisciplinary Journal of Philosophy. https://doi.org/10.1080/0020174X.2021.1899044.

Wenmackers, S., \& Horsten, L. (2013). Fair infinite lotteries. Synthese, 190(1), 37-61.

Wilsch, T. (2016). The deductive-nomological account of metaphysical explanation. Australasian Journal of Philosophy, 94(1), 1-23. https://doi.org/10.1080/00048402.2015.1033637. 
Zimmerman, D. (2007). The privileged present: Defending an "a-theory" of time. In T. Sider, J. Hawthorne, \& D. W. Zimmerman (Eds.), Contemporary Debates in Metaphysics (pp. 211-225). Blackwell.

Zylstra, J. (2019). Making semantics for essence. Inquiry: An Interdisciplinary Journal of Philosophy, $62(8), 859-876$.

Publisher's Note Springer Nature remains neutral with regard to jurisdictional claims in published maps and institutional affiliations. 